\title{
ON A CLASS OF UNIMODAL DISTRIBUTIONS
}

\author{
R. G. LAHA ${ }^{1}$
}

A distribution function $F(x)$ is said to be unimodal [1, p. 157] if there exists at least one value $x=a$ such that $F(x)$ is convex for $x<a$ and concave for $x>a$. The point $x=a$ is called the vertex of the distribution. In particular, if $F(x)$ is absolutely continuous, then the corresponding probability density function $p(x)=F^{\prime}(x)$ is nondecreasing for $x<a$ and nonincreasing for $x>a$. In the present paper we shall establish the unimodality of a class of distribution functions which were studied by Linnik in [2]. He has shown that for any real $\alpha$ in the interval $0<\alpha \leqq 2$ the function

$$
f(t)=\frac{1}{1+|t|^{\alpha}}
$$

is the characteristic function of a symmetric and absolutely continuous distribution function. We denote this class of distribution functions by $C$. We shall now prove the following theorem.

TheOREM. Every distribution function belonging to the class $C$ is unimodal.

For the proof of this theorem we give first the following lemma which may be of some independent interest.

LEMMA 1. Let $f(t)$ be a continuous real-valued and even function of the real variable $t$ such that $f(0)=1$ and $f(t)=A(t)$ for $t>0$ where the function $A(t)$ satisfies the following conditions:

(i) $A(z)$ defined as a function of the complex variable $z\left(z=r e^{i \theta}=t+i v\right.$, $t$ and $v$ both real) is regular in the region $D\left(r>0 ;-\epsilon_{1}<\theta<\pi / 2+\epsilon_{2}\right.$ where $\epsilon_{1}$ and $\epsilon_{2}$ are arbitrary small positive numbers) of the complex z-plane;

$$
\begin{array}{cl}
|A(z)|=O(1) & \text { as }|z| \rightarrow 0, \\
|A(z)|=O\left(|z|^{-i}\right) & \text { as }|z| \rightarrow \infty \quad(\delta>1) ; \\
\operatorname{Im} A(i v) \leqq 0 & \text { for } v>0 .
\end{array}
$$

Then $f(t)$ is the characteristic function of a symmetric unimodal and absolutely continuous distribution function.

Received by the editors January 18, 1960 and, in revised form, May 2, 1960.

${ }^{1}$ This work was supported in part by The National Science Foundation Grant NSF-G-4220 and in part by the Office of Naval Research Contract NR-042-064. 
Proof. First we note that for real values of $t$

$$
\begin{array}{ll}
|f(t)|=O(1) & \text { as }|t| \rightarrow 0, \\
|f(t)|=O\left(|t|^{-\delta}\right) & \text { as }|t| \rightarrow \infty,
\end{array}
$$

so that $f(t)$ is absolutely integrable over the interval $(-\infty<t<+\infty)$. Therefore for any real $x$ the function

$$
p(x)=\frac{1}{2 \pi} \int_{-\infty}^{\infty} e^{-i t x} f(t) d t=\frac{1}{\pi} \int_{0}^{\infty} \cos t x f(t) d t
$$

exists and is a continuous, real-valued and even function of $x$ so that we have $p(x)=p(-x)$ for $x \geqq 0$. We shall now show that $p(x) \geqq 0$ for $x \geqq 0$. We see easily from (1) that for $x \geqq 0$

$$
p(x)=\frac{1}{\pi} \operatorname{Re} \int_{0}^{\infty} e^{i t x} f(t) d t=\frac{1}{\pi} \operatorname{Re} \int_{0}^{\infty} e^{i t x} A(t) d t .
$$

In order to evaluate the integral on the right-hand side of (2), we consider the function $\psi(z)=e^{i z x} A(z)$ ( $z$ complex and $x \geqq 0$ ) and a closed contour $\Gamma$ in the first quadrant of the complex $z$-plane $(z=t+i v$, $t$ and $v$ both real) consisting of the real axis from $\rho$ to $R$, the larger circular arc of radius $R$, the imaginary axis from $i R$ to $i \rho$ and finally the smaller arc of radius $\rho$. The function $\psi(z)$ is regular in the contour $\Gamma$. Therefore, according to the theorem of Cauchy we have

$$
\int_{\Gamma} e^{i z x} A(z) d z=0
$$

or

$$
\int_{\rho}^{R} e^{i t x} A(t) d t+I+\int_{R}^{\rho} e^{-v x} A(i v) i d v+J=0 .
$$

Here $I$ and $J$ denote respectively the integrals along the circular arcs of radii $R$ and $\rho$. We can easily verify that $I \rightarrow 0$ as $R \rightarrow \infty$ and similarly $J \rightarrow 0$ as $\rho \rightarrow 0$. Therefore as $\rho \rightarrow 0$ and $R \rightarrow \infty$, we obtain from (3)

$$
\int_{0}^{\infty} e^{i t x} A(t) d t=i \int_{0}^{\infty} e^{-v x} A(i v) d v .
$$

Then we conclude easily from (2) and (4) that for $x \geqq 0$

$$
p(x)=-\frac{1}{\pi} \int_{0}^{\infty} e^{-v x}[\operatorname{Im} A(i v)] d v .
$$

The non-negativity of $p(x)$ for $x \geqq 0$ follows immediately from the condition (iii) of the lemma. Finally we show that for $x \geqq 0$, the 
probability density function $p(x)$ is a decreasing function of $x$. From (5) we see that for $x_{1}>x_{2}>0$,

$$
p\left(x_{1}\right)<p\left(x_{2}\right)<p(0)
$$

so that the probability density function $p(x)$ has a unique maximum at the point $x=0$. This completes the proof of the lemma.

Proof of The Theorem. For the proof of the theorem, we shall discuss separately three mutually exclusive possible cases:

CASE $1(0<\alpha \leqq 1)$. In this case we consider the function

$$
\theta(t)=f(t)+t f^{\prime}(t)=\frac{1+(1-\alpha)|t|^{\alpha}}{\left(1+|t|^{\alpha}\right)^{2}} .
$$

We can easily verify after some elementary computations that for $t>0$

$$
\theta^{\prime \prime}(t)=\frac{\alpha t^{\alpha-2}}{\left(1+t^{\alpha}\right)^{4}}\left[\left(1-\alpha^{2}\right)+2\left(1+2 \alpha^{2}\right) t^{\alpha}+\left(1-\alpha^{2}\right) t^{2 \alpha}\right]>0 .
$$

Thus we conclude that the function $\theta(t)$ introduced in (6) is a continuous, real-valued and even function of the real variable $t$ and further it is convex for $t>0$ and $\theta(0)=1$. The function $\theta(t)$ satisfies all the conditions of Pólya's theorem [3, p. 116] and hence it is a characteristic function. Finally we note that the function $f(t)$ can be represented in the form

$$
f(t)=\frac{1}{t} \int_{0}^{t} \theta(u) d u
$$

where $\theta(u)$ itself is a characteristic function. The unimodality of this class of distribution functions follows immediately from the theorem due to Khintchine $[1$, p. 160].

CASE $2(1<\alpha<2)$. Here we define the function

$$
A(z)=\frac{1}{1+z^{\alpha}}
$$

as a function of the complex variable $z$ and then verify easily that $A(z)$ satisfies the conditions (i), (ii) and (iii) of Lemma 1. Therefore the unimodality of this class follows as a direct consequence of this lemma.

CASE $3(\alpha=2)$. In this case it is well known that the probability density function of the corresponding distribution function is given by

$$
p(x)=\frac{1}{2} e^{-|x|}, \quad(-\infty<x<\infty),
$$


which has a unique maximum at the point $x=0$. This completes the proof of the theorem. The following corollary is a direct consequence of this theorem.

Corollary. Every symmetric stable distribution function is unimodal.

This result has been already proved by Wintner [4, p. 33]. We give below a simple alternative proof. First we state two lemmas:

LEMмA 2. The convolution of two symmetric unimodal distribution functions is symmetric unimodal.

This lemma is due to Wintner [4, p. 30].

LEMMA 3. If a sequence of unimodal distribution functions converges to a distribution function, then the limiting distribution function is also unimodal.

The proof of this lemma is given in [1, p. 160].

We now turn to the proof of the corollary. First, we note that the characteristic function of a symmetric stable distribution function is given by $g(t)=e^{-|\varepsilon|^{\alpha}}(0<\alpha \leqq 2)\left[1\right.$, p. 164]. We write $a_{n}=n^{-1 / \alpha}$ where $n$ is a positive integer. Then we conclude from our theorem and Lemma 2 that for every positive integer $n$, the function

$$
g_{n}(t)=\left[f\left(a_{n} t\right)\right]^{n}=\frac{1}{\left(1+\frac{|t|^{\alpha}}{n}\right)^{n}}
$$

is the characteristic function of a symmetric unimodal distribution function. The proof of the corollary follows immediately from Lévy's continuity theorem and Lemma 3.

\section{REFERENCES}

1. B. V. Gnedenko, and A. N. Kolmogorov, Limit distributions for sums of independent random variables (English translation by $\mathrm{K}$. L. Chung), Reading, Massachusetts, Addison-Wesley Publishing Co., Inc., 1954.

2. Yu. V. Linnik, Linear forms and statistical criteria. Part 2 (in Russian), Ukrain. Mat. Z. vol. 5 (1953) pp. 247-290.

3. G. P6lya, Remarks on characteristic functions, Proceedings of the First Berkeley Symposium, 1949, pp. 115-123.

4. A. Wintner, Asymptotic distributions and infinite convolutions, lecture notes, Institute for Advanced Study, 1938.

Catholic University and

CORNELl University 